\title{
The Contribution of Radiology Service Staffs in the Optimization of TAP-CT Doses for Cancer Patients: A Comparative Study of Two Hospitals in Northern Morocco
}

\author{
Bougana Ihsane $^{1}$, Benabdelouahab Farid ${ }^{1}$, Kacemi Loubna ${ }^{2}$ \\ ${ }^{1}$ Laboratory of Materials and Radiations Physics, Department of Physics, Faculty of Sciences, University Abdelmalek Essaadi, Tetouan, \\ Morocco \\ ${ }^{2}$ Division of Radiology and Medical Imagery, Hospital Mohammed VI, M'diq, Morocco
}

\section{Email address:}

ihssanebougana@hotmail.com (B. Ihsane)

\section{To cite this article:}

Bougana Ihsane, Benabdelouahab Farid, Kacemi Loubna. The Contribution of Radiology Service Staffs in the Optimization of TAP-CT Doses for Cancer Patients: A Comparative Study of Two Hospitals in Northern Morocco. Journal of Cancer Treatment and Research. Vol. 9, No. 4, 2021, pp. 56-62. doi: 10.11648/j.jctr.20210904.11

Received: March 13, 2021; Accepted: May 6, 2021; Published: October 12, 2021

\begin{abstract}
The increasing share of Thoraco-Abdomino-Pelvic-CT scan (TAP-CT) dose delivered to cancer patients requires particular vigilance. In fact, the radioprotection practices of our cancer patients are poorly respected, especially in terms of the number of acquisitions performed by practitioners. For instance, when performing a TAP-CT scan in cancer patients, the series without injection and the series with injection include arterial time, portal time, and rarely late time, lead to three to four acquisitions. Most practitioners do this routinely without considering whether these acquisitions are justified or not. This work assesses the practices carried out in the service of radiology in two hospitals in the province of Tetouan (northern Morocco). The overall purpose is to improve the radioprotection of our cancer patients. The retrospective investigation involved a total of 100 patients performed TAP examination. The $\mathrm{PDL}_{\text {total }}$ is in the order of $500.72 \pm 15.08 \mathrm{mGy} . \mathrm{cm}$, and the effective dose (E) is of the order of $7.51 \pm 0.226 \mathrm{mSv}$. Sex and ages variables did not show any significant differences according to t-test and ANOVA respectively. However, the variable "number of acquisitions" per examination showed a significant difference for $\mathrm{PDL}_{\text {total }}$ and the Effective Dose $(\mathrm{F}=16.462 ; \mathrm{p}<0.001)$. The MANOVA analysis showed that the variables gender and number of acquisitions showed a significant effect; $\left(D_{\text {gender }}=0.748 ; p=0.042\right)$ and $\left(D_{\text {number of acquisitions }}=11.888 ; p<0.001\right)$. By comparing the results of two hospitals, we found a large variation in the delivered doses. The radiologist himself seems to be a significant factor that can influence unnecessary acquisitions and therefore the total delivered dose. Consequently, the standardization of TAP protocols and the sharing of best practices between hospitals becomes a necessary approach towards dose optimization.
\end{abstract}

Keywords: Cancer Patient, Dose, TAP-CT Scan, Dosimetry, Optimization

\section{Introduction}

In recent decades, with a perspective to reduce the radiation dose associated with the scanners, new technologies have been made available to practitioners [1], such as tube current modulation (TCM) [1-5] and iterative reconstructions (IR) [6]. However, these technological advances revealed to be insufficient to optimize the radiation doses associated with the scanner. Indeed, in 1921, Marie Curie stated "Whatever the value of equipment and methods, it is the personnel responsible for their use that ultimately depends on effective performance. The X-ray equipment must be handled by expert hands, and the methods must be applied intelligently" [7]. This statement bring our intention to the contribution of radiological staff in the optimization process.

In this context, recent studies by a French team leading by Gervaise A. have assessed the influence of the staff behavior on reducing the radiation associated with the TAP scanner. In this paper, we will illustrate the results of two of his relevant published studies:

The first study, published in 2013 by [8], was carried out on 84 patients suspected of multiple trauma. They received whole body examinations by CT scan, with a follow-up of one year. This study analyzed the number of acquisitions - dose 
relationship. Their results showed a 50\% dose reduction for the acquisition of a single series in spontaneous contrast in the portal phase versus two identical acquisitions without and after injection in the portal phase.

The second study aimed to show that acquisition limitation is a simple way to reduce the dose to our patients. Published in 2016 [9], this study have included 365 patients performed an Abdomino-pelvic scanner. The study compared between three methods: method 1 (M1) using kidney storytellers, method 2 (M2) using the lower edge of the T10 vertebra, and method 3 (M3) using the insertion point between the left diaphragmatic dome and the anterior border of the vertebral bodies (Figure $1 \mathrm{a}, \mathrm{b}, \mathrm{c}$ ). The study concluded an average reduction in acquisition length of $20.5 \%$ for method 1 (M1), therefore a reduction in the dose.

Thus, the results of these studies suggest the influence of behavioral factors on dose optimization.

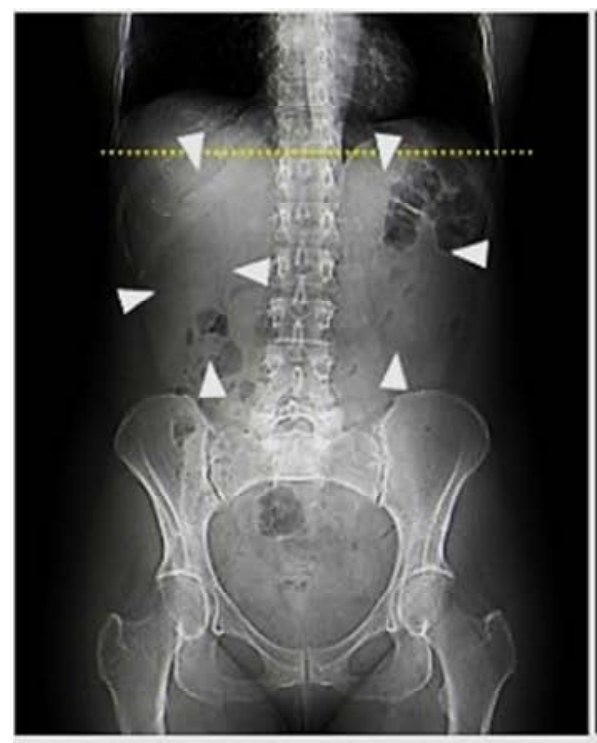

(a)

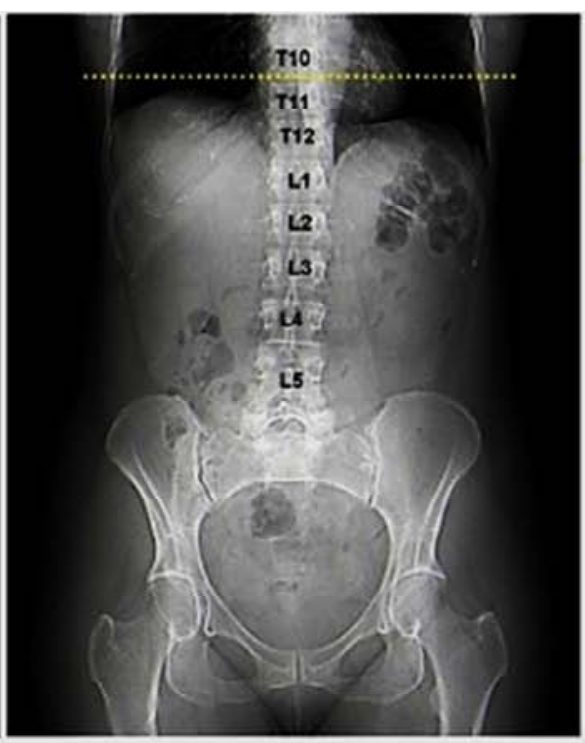

(b)

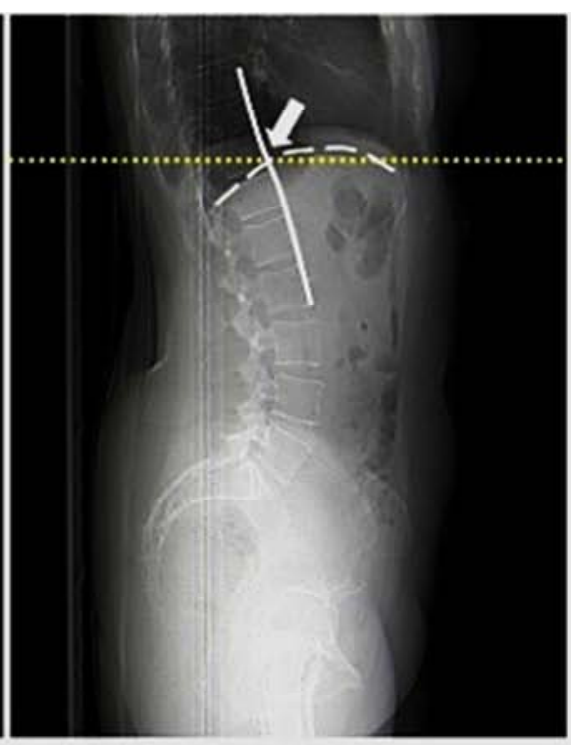

(c)

Figure 1. M1 (a) the upper limit of acquisition was placed by locating the top of the kidneys using the renal contours as a reference (arrowheads). M2 (b) used the bottom margin of T10 as a reference. M3 (c) used the image of the side scout and the point of intersection (arrow) between the anterior margin of the vertebral bodies (solid line) and the left diaphragmatic dome (dotted line) [9].

This work investigates the importance of radiological staff behaviors in optimizing the doses delivered to our patients. Our study presents a clear vision of the essential role of radiology staffs - radiologists in particular - in optimizing the doses received by patients. To realize our vision, we conducted a comparative study with the results of our previous article [10]. For comparative purposes, we followed the same methodology as in our previous work.

\section{Material and Methods}

\subsection{Dosimetric Calculation Method}

The approach used to calculate the dose delivered to our patients during a Thoraco-Abdomino-Pelvic scanner is described in [10].

\subsection{Approaches Followed}

This approach consists of estimating the effective dose based on conversion factors $\left(\mathrm{E}_{\mathrm{pdl}}\right)$ according to the anatomical region and age (Table 1) [11]. In this approach, the exam $\mathrm{PDL}_{\text {total }}$ was multiplied by the irradiated region- specific $\mathrm{E}_{\mathrm{pdl}}$ to estimate the effective dose. The exam $\mathrm{PDL}_{\text {total }}$ displayed on the scanner console allows easy estimation of the effective dose [12].

\subsection{Measures Used in Our Study}

Two main dosimetric measures reflecting the irradiation delivered by the scanner were used in this study:

Product dose length (PDL) as previously studied [10]. At the end of the examination, we noted directly the PDL value for each acquisition series carried out during the examination. The total dose $\left(\mathrm{PDL}_{\text {total }}\right)$ associated with the TAP examination then corresponds to the sum of the doses received at each acquisition (Figure 2)

Effective dose E [10], the calculation of the effective dose for our sample is based on the formula retrieved from [11:

$$
\mathrm{E}=\mathrm{E}_{\mathrm{pdl}} \times \mathrm{PDL}_{\text {total }}
$$

We thus calculate, for the TAP CT scan, the corresponding effective dose to which each of the patients in our sample is exposed. 


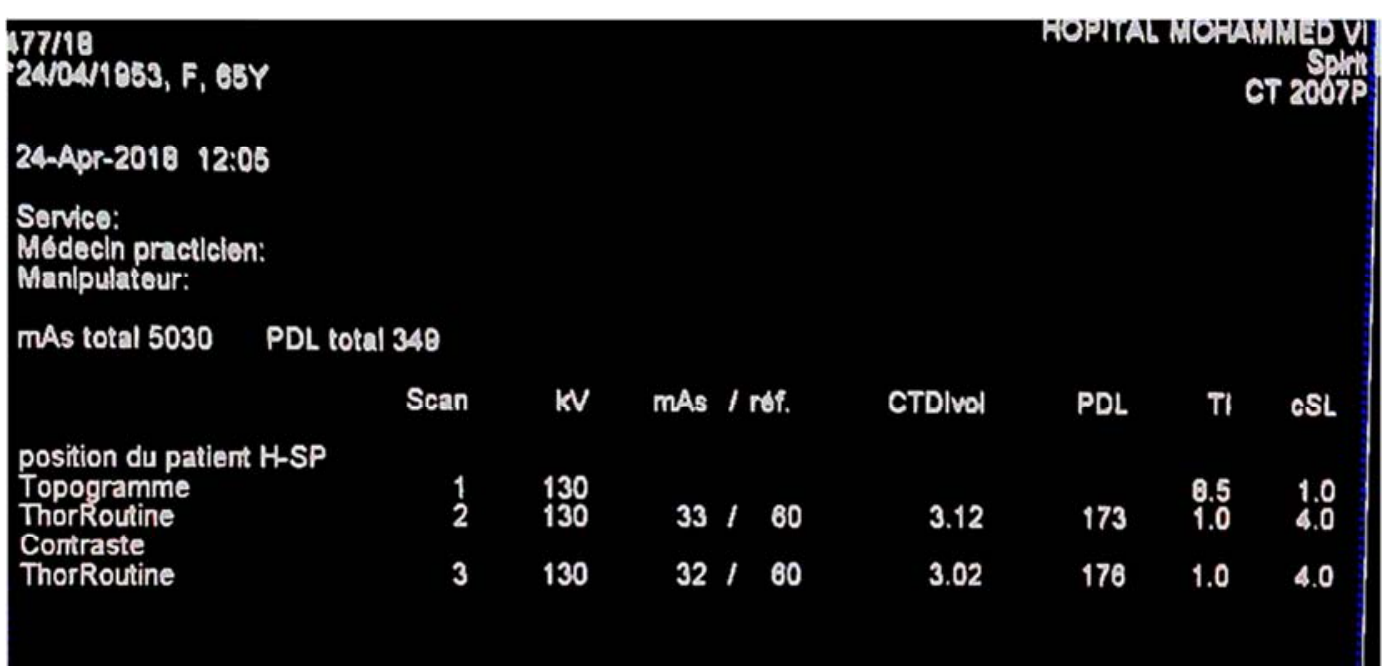

Figure 2. Extract from the report of the dose delivered on the TAP monitor (PDL $L_{\text {total }}$ : 349 on two acquisitions) for a 65-year-old woman.

\subsection{The Studied Population}

This investigation took place between 2017-2018, in the medical imaging department of the M'diq hospital, equipped with a double-bar Siemens Somatom Spirit 2007 scanner. The scanner used had the possibility of helical acquisition as well as a means of reducing the CARE DOSE dose. Our survey is made up of 100 patients, $57 \%$ of whom are women and $56 \%$ are between 41 and 60 years old (Table 1). Information on these patients was extracted from the digital scanner system. Our patients underwent TAP CT for the cancer-staging objective using a fixed potential of $130 \mathrm{KV}$, with a mAs load range from 26 to $62 \mathrm{mAs}$ and $\mathrm{mAs}_{\text {ref }}$ varied from 60 to 90 . The numbers of acquisitions varied between 1 and 5 , but the majority ( $81 \%)$ had 1 to 2 acquisitions.

Table 1. Detailed descriptions of the population studied according to PDLtotal and the Effective Dose.

\begin{tabular}{lllll}
\hline Descriptive & & & Effective Dose in mSv \pm SE & PDL $_{\text {total }}(\mathbf{m G y . c m}) \pm$ SE \\
\hline Mean of sample & & $\mathrm{N}=100$ & $7,51 \pm 0,226$ & $500,72 \pm 15,08$ \\
& $<30$ & 5 & $6,69 \pm 0,611$ & $446,40 \pm 40,79$ \\
Age & {$[31-40]$} & 9 & $7,33 \pm 0,805$ & $489,00 \pm 53,68$ \\
& {$[41-60]$} & 56 & $7,72 \pm 0,271$ & $515,30 \pm 18,11$ \\
\multirow{3}{*}{ Gender } & $>60$ & 30 & $7,29 \pm 0,499$ & $486,06 \pm 33,31$ \\
& $\mathrm{~F}$ & 57 & $7,60 \pm 0,274$ & $506,85 \pm 18,30$ \\
\multirow{4}{*}{ Number of acquisition } & $\mathrm{M}$ & 43 & $7,38 \pm 0,382$ & $492,58 \pm 25,52$ \\
& & & & \\
& {$[1-2]$} & 81 & $6,98 \pm 0,227$ & $465,96 \pm 15,18$ \\
& 3 & 14 & $9,24 \pm 0,322$ & $616,34 \pm 21,52$ \\
\end{tabular}

\section{Results}

\subsection{Evaluation of PDL $L_{\text {total }}$ and Effective Dose (E)}

The total absorbed dose per exam or $\mathrm{PDL}_{\text {total }}$ is in the order of $500.72 \pm 15.08 \mathrm{mGy} . \mathrm{cm}$. While the effective dose (E) is of the order of $7.51 \pm 0.226 \mathrm{mSv}$ (Table 1).

The distribution of these values according to the sex variable shows an average difference in $\mathrm{PDL}_{\text {total }}$ within $+14.27 \mathrm{mGy} . \mathrm{cm}$ and an average difference of the effective doses of the order of $+0.214 \mathrm{mSv}$ in favor of "Female". However, these differences remain non-significant for the two variables $(\mathrm{F}=0.467 ; \mathrm{p}=0.642)$.

Considering the age variable, the ANOVA test showed a non-significant difference between the four age groups for the two variables total PDL and effective dose $(\mathrm{F}=0.496$; $\mathrm{p}=0.686$ ) (Table 2).
Table 2. ANOVA followed by Tukey post-hoc test for the variables Total PDL and Effective dose versus age variable.

\begin{tabular}{|c|c|c|c|}
\hline \multirow{2}{*}{$\begin{array}{l}\text { Tukey Post- } \\
\text { hoc test }\end{array}$} & \multirow{2}{*}{ Age Classes } & \multirow{2}{*}{$\mathbf{N}$} & sub-group for alpha $=0.05$ \\
\hline & & & 1 \\
\hline \multirow{5}{*}{$\begin{array}{l}\text { PDL total } \\
\text { (mGy.cm) }\end{array}$} & $<30$ & 5 & 446,400 \\
\hline & $>60$ & 30 & 486,066 \\
\hline & {$[31-40]$} & 9 & 489,000 \\
\hline & [41-60] & 56 & 515,303 \\
\hline & Significance & & 0,711 \\
\hline \multirow{5}{*}{$\begin{array}{l}\text { Effective Dose } \\
(\mathrm{mSv})\end{array}$} & $<30$ & 5 & 6,696 \\
\hline & $>60$ & 30 & 7,291 \\
\hline & {$[31-40]$} & 9 & 7,335 \\
\hline & [41-60] & 56 & 7,729 \\
\hline & Significance & & 0,711 \\
\hline
\end{tabular}

Whereas, the variable "number of acquisitions" per examination showed a significant difference for PDLtotal and for the Effective Dose $(\mathrm{F}=16.462 ; \mathrm{p}<0.001)$. Turkey post-hoc test showed that this significant difference is particularly 
evident between the TAP examination with 1 to 2 acquisitions and the examination with more than 2 acquisitions (Table 3).

Table 3. Tukey post-hoc test for the number of acquisitions.

\begin{tabular}{lllll}
\hline \multirow{2}{*}{$\begin{array}{l}\text { Number of } \\
\text { acquisition }\end{array}$} & $\mathbf{N}$ & \multicolumn{2}{l}{ PDL $_{\text {Total }}(\mathbf{m G y . c m )}$} & \multicolumn{2}{c}{ Effective Dose (mSv) } \\
\cline { 2 - 5 } & & Sous-ensemble pour alpha=0.05 & Sub-group for alpha=0.05 \\
\hline$[1-2]$ & 81 & 465,96296 & $\mathbf{2}$ & $\mathbf{1}$ \\
3 & 14 & & 616,98944 & $\mathbf{2}$ \\
4 or more & 5 & & 739,40000 & 9,24857 \\
Significance & & 1 &, 086 & 11,09100 \\
\hline
\end{tabular}

\subsection{Multivariate Analysis}

In order to test the effect of the interaction between the independent variables of this study on total PDL and the effective dose received by the patients in our sample, the MANOVA multivariate analysis was used.

Table 4 shows the effect of the inter-subject interaction of the variables: age, sex and the number of acquisitions on the variables $\mathrm{PDL}_{\text {total }}$ and Effective Dose. The coefficients of determination $\mathrm{R}^{2}$ for the two variables are acceptable and significant for the two dependent variables: $R^{2}=0.350$ (adjusted $\mathrm{R}^{2}=0.252$ ). We clearly note that the variables gender and number of acquisitions showed a significant effect; $\quad\left(D_{\text {gender }}=0.748 ; \quad \mathrm{p}=0.042\right)$ and $\quad\left(D_{\text {number }}\right.$ of acquisitions $=11.888 ; \mathrm{p}<0.001)$.

Table 4. Multivariate Analysis for PDL total and Effective Dose.

\begin{tabular}{|c|c|c|c|c|}
\hline Independent variables & Dependent variables & ddl & D & Sig. \\
\hline \multirow{2}{*}{ Gender } & $\mathrm{PDL}_{\text {total }}(\mathrm{mGy} . \mathrm{cm})$ & 1 & 4,256 & 0,042 \\
\hline & Effective dose (mSv) & 1 & 4,256 & 0,042 \\
\hline Age groups & $\mathrm{PDL}_{\text {total }}(\mathrm{mGy} . \mathrm{cm})$ & 3 & 0,748 & 0,526 \\
\hline \multirow{2}{*}{ Number of acquisition } & $\mathrm{PDL}_{\text {total }}(\mathrm{mGy} . \mathrm{cm})$ & 2 & 11,888 & 0,000 \\
\hline & Effective dose (mSv) & 2 & 11,888 & 0,000 \\
\hline Gender * Age groups & $\mathrm{PDL}_{\text {total }}(\mathrm{mGy} . \mathrm{cm})$ & 3 & 0,306 & 0,821 \\
\hline \multirow{2}{*}{ Gender * Number of acquisition } & $\mathrm{PDL}_{\text {total }}(\mathrm{mGy} . \mathrm{cm})$ & 0 & . & . \\
\hline & Effective dose (mSv) & 0 & . & . \\
\hline \multirow{2}{*}{ Age groups $*$ Numbre of acquisition } & $\mathrm{PDL}_{\text {total }}(\mathrm{mGy} . \mathrm{cm})$ & 4 & 1,904 & 0,117 \\
\hline & Effective dose (mSv) & 4 & 1,904 & 0,117 \\
\hline \multirow{3}{*}{ Gender * Age groups * Number of acquisition } & $\mathrm{PDL}_{\text {total }}(\mathrm{mGy} . \mathrm{cm})$ & 0 & . & . \\
\hline & Effective dose (mSv) & 0 & . & . \\
\hline & $\mathrm{PDL}_{\text {total }}(\mathrm{mGy} . \mathrm{cm})$ & 99 & & \\
\hline
\end{tabular}

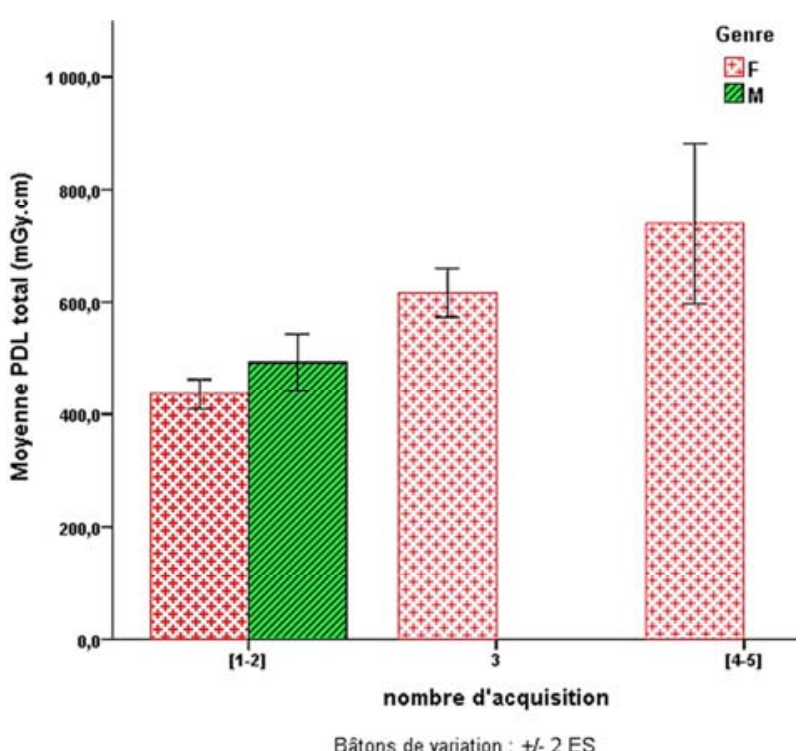

Figure 3. Distribution of acquisition number per gender.

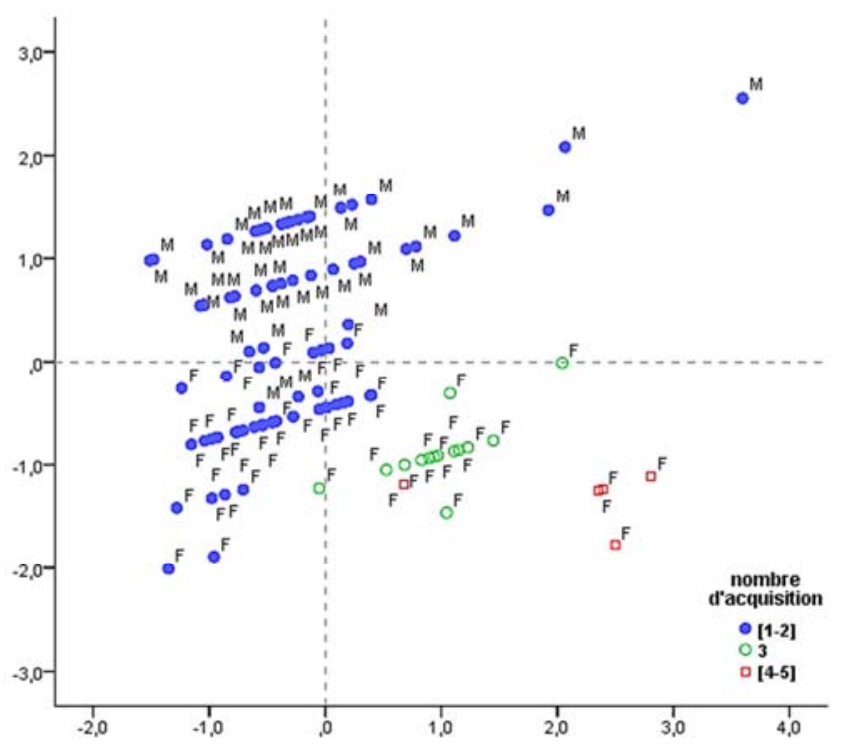

Figure 4. Scatter plots of number of acquisition by Gender. 
However, the combined effect of the latter alone and/or with the age class variable is not at all significant. It should also be noted that the combination of the variables gender and number of acquisitions does not give any significant results; this is due to the fact that the male patients in our sample only received 1 to 2 acquisitions, while the female patients received in addition 3, 4 and 5 acquisitions (Figure 3 and Figure 4).

The sensitivity of practitioners to the radiation protection of patients can be achieved using diagnostic reference levels (DRLs) which allow a comparison of a particular practice with a reference. A Belgian multicenter study [13] investigated the request for medical imaging exams with focus on three frequently requested examinations, CT of the lumbosacral spine, abdominal CT, and Thoraco-Abdomino-pelvic CT (TAP-CT). The data for this study was collected from eight hospitals, each one work with different scanner devices. The study showed a $\mathrm{PDL}_{\text {median }}$ of 691 mGy.cm with $95 \%$ confidence interval $\mathrm{CI}=[556-859]$. If compared these values with those found by [14], $\mathrm{PDL}_{\text {median }}=756 \mathrm{mGy} . \mathrm{cm},[13]$ concluded that there was no significant difference within $95 \%$ IC.
A Scottish study by [15] has aimed to determine whether the NRD (940 mGy.cm), established in 2003 in the United Kingdom (UK), is still appropriate. The results indicated that the doses of TDM-TAP are lower than those previously reported (median $800 \mathrm{mGy} . \mathrm{cm}, 75^{\text {th }}$ percentile $840 \mathrm{mGy} . \mathrm{cm}$ ).

In this study, the PDL at the $75^{\text {th }}$ percentile is $573 \mathrm{mGy} . \mathrm{cm}$, which shows a decrease of $78.64 \%$ compared to our first study [10]; while it is lower by $52.96 \%$ compared to the Tunisian study [16]. Also, it shows a reduction of $17.07 \%$ compared to the Belgian study [13] and a decrease of $31.87 \%$ compared to the Scottish study [15]. Furthermore, the PDL at the $75^{\text {th }}$ percentile is lower in $42.7 \%$ of cases compared to the French NRDs (1000mGy.cm) (Figure 5) and comparable to the French NRDs 2015 (771 mGy.cm) (Figure 6).

The possible explanation for this drop in our result is attributed to:

The presence of a radiologist during the TAP examination controlling the unnecessary acquisitions;

The hospital has a device equipped with a reduction technique (CAREDOSE).

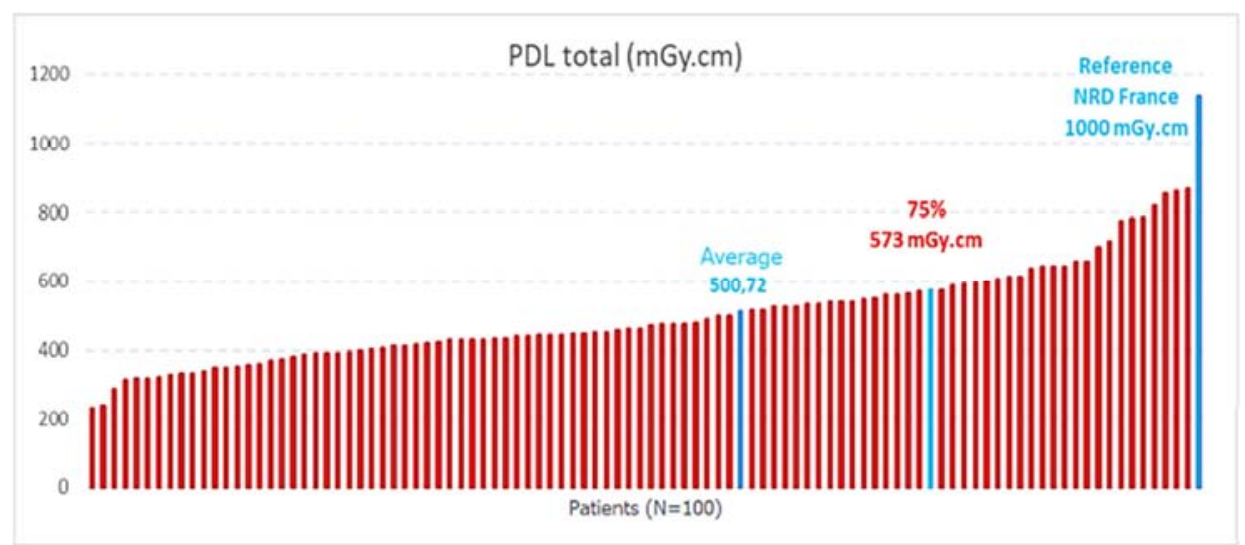

Figure 5. Distribution of the dose-length (DLP) product values by TAP scanner examination, comparing the $75^{\text {th }}$ percentile value with the French NRDs.

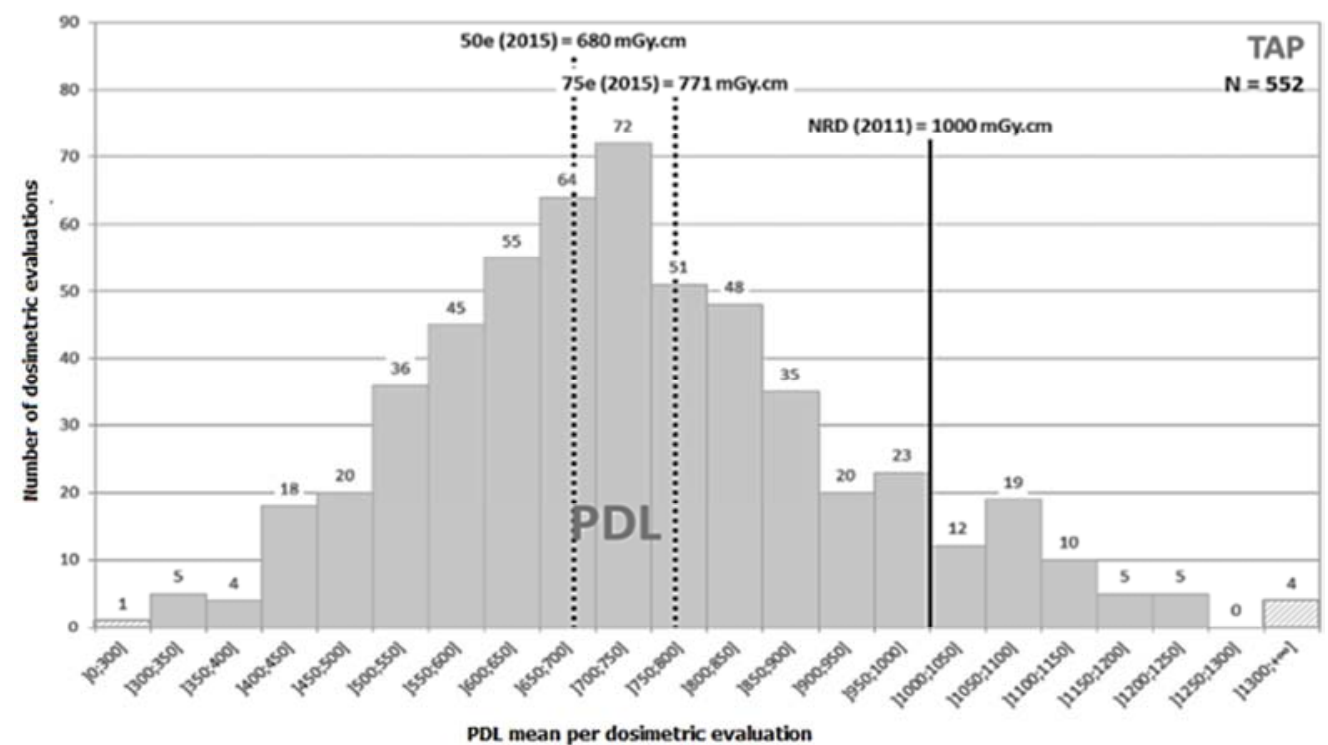

Figure 6. Distribution of mean PDL values resulting from dosimetric evaluations carried out for the examination of the thoraco-abdomino-pelvic region [20]. 
For the effective dose received by our patients, the international references [17] recommend an effective dose for a TAP scanner of the order of $10 \mathrm{mSv}$ per examination. Thus, patients who perform a CT scan every 6 months receive an effective dose close to the annual legal limit for workers in exposed nuclear centers (of the order of $20 \mathrm{mSv}$ ). Considering the age of the patient, the younger he/she irradiated, the greater the risk of radio-induced secondary cancer [17]. The results obtained in our study show an average effective dose of $7.51 \pm 0.226 \mathrm{mSv}$ per examination. Which is within the standards of international references of the effective dose $[18,19,11]$. Thus, the average effective dose for the studied age groups remains within the range of international standards and varies between $6.696 \pm 0.611$ for the $<30$ years old class and $7.729 \pm 0.271$ for the [41-60] class (Table 5). However, by number of acquisitions, this effective dose exceeds these standards when the number of acquisitions is greater than or equal to three acquisitions (Table 6). Therefore, reducing the number of acquisitions remains a very important parameter in reducing the risk of a radiological effect.

Finally, updating the NRDs for the scanner and in particular for the TAP exam has become a major necessity. In this context, the analysis of the dosimetric data sent to IRSN by the radiology centers shows a reduction in the doses delivered to patients by $\mathrm{CT}$ scan, with an average reduction of $15 \%$ [20]. This led IRSN to recommend a downward revision of the NRD values for adults.

Table 5. Effective doses and 95\% Confidence Interval (CI) by age group.

\begin{tabular}{|c|c|c|c|c|}
\hline Age groups & $<30$ & {$[31-40]$} & {$[41-60]$} & $>60$ \\
\hline $\begin{array}{l}\text { Dose Total Effective ( } \mathrm{mSv} \text { ) for a unique TAP exam } \\
\text { IC } 95 \%\end{array}$ & $\begin{array}{l}6,696 \pm 0,611 \\
{[4,99-8,39]}\end{array}$ & $\begin{array}{l}7,335 \pm 0,805 \\
{[5,47-9,19]}\end{array}$ & $\begin{array}{l}7,729 \pm 0,271 \\
{[7,18-8,27]}\end{array}$ & $\begin{array}{l}7,291 \pm 0,499 \\
{[6,26-8,31]}\end{array}$ \\
\hline
\end{tabular}

Table 6. Effective doses and 95\% Confidence Interval (CI) by number of acquisitions.

\begin{tabular}{llll}
\hline Number of acquisitions & {$[\mathbf{1 - 2}]$} & $\mathbf{3}$ & $\mathbf{4}$ ou plus \\
\hline Dose Total Effective $(\mathrm{mSv})$ for unique TAP exam & $6,98 \pm 0,227$ & $9,24 \pm 0,322$ & $11,09 \pm 1,070$ \\
IC $95 \%$ & {$[6,536-7,442]$} & {$[8,551-9,946]$} & {$[8,119-14,062]$}
\end{tabular}

\section{Conclusion}

The justification of the number of acquisitions remains the simplest and most relevant tool when we want to control the doses delivered to our patients. The role of the radiologist is essential in this justification. Developments in CT technology have a clear impact on the radiation protection of patients, but just using these technological advances without smart hands does not systematically reduce doses.

The practical recommendations drawn from this study:

1. The presence of the radiologist is mandatory during TAP exams

2. Significant variations in TAP practices and protocols between the two hospitals so we recommend Standardization of TAP protocols

3. Sharing of best practices between imaging centers

4. Variations in practices according to technicians and radiologists

5. Get the acquisition right the first time

6. Act on behavioral factors

7. Update of DRLs, $25^{\text {th }}$ percentile instead of $75^{\text {th }}$ percentile.

\section{References}

[1] Gervaise A., (2016) Optimization and reduction of the radiation dose by scanner: technical aspects and impact in current clinical practice. Doctoral thesis defended on 03-102016 at the University of Lorraine.
[2] Kalra MK., Maher MM., (2004) Technique and applications of automatic tube current modulation for CT. Radiology. DOI: 10.1148/radiol.2333031150

[3] Soderberg M., Gunnarsson M., (2010) Automatic exposure control in Computed tomography an evaluation of système from different manufacteurers. Acta Radiol. doi: $10.3109 / 02841851003698206$.

[4] Rampado O., Marshisio F., Izzo A., (2009) Effective dose and image quality evaluations of an automatic CT tube current modulation system with an anthropomorphie phantom. Eur J Radiol. doi: 10.1016/j.ejrad.2008.06.027.

[5] Raman SP., Johnson PT., (2013) CT dose reduction applications: avaible tools on the latest generation of CT scanners. J Am Coll Radiol. doi: 10.1016/j.jacr.2012.06.025.

[6] Macri F., (2017) Optimization of acquisition and reconstruction parameters for dose reduction in computed tomography in the diagnostic assessment of chest pain in emergency rooms. HAL Id: tel 01508840 (https://tel.archivesouvertes.fr/tel-01508840).

[7] Marie Curie (1921) La radiologie et la guerre, page 99. Félix Alcan. Prais https://gallica.bnf.fr/ark:/12148/bpt6k67970j/f2.image.

[8] Gervaise A., Naulet P., (2013) Évaluation de l'intérêt de l'acquisition abdomino-pelvienne sans injection lors de la réalisation d'un scanner corps entier chez un patient suspect de polytraumatisme. Journal de Radiologie Diagnostique et Interventionnelle, Volume 94, Issue 4, Pages 424-432.

[9] Gervaise, A., Teixeira P. (2016) Optimizing $z$-axis coverage of abdominal CT scans of the urinary tract: a proposed alternative proximal landmark for acquisition planning. Br J Radiol DOI: 10.1259/bjr.20160197. 
[10] Bougana I., Benabdelouahab A., Loutfi A., El Ouahrani A. (2018) Optimization with reduction of patient exposure during CT/TAP scans: case of cancer patients in northern Morocco. Radioprotection. $\quad 53 \quad$ (2): $115-112$. https://doi.org/10.1051/radiopro/2018014.

[11] Delchambre-Roussel M. 2012. Calcul de la dose efficace cumulée reçue par nos patients en tomodensitométrie diagnostique: création d'un outil informatique pour le médecin généraliste. Human Health Pathol. https://dumas.ccsd.cnrs.fr/dumas00745007/document.

[12] Menzel HG, Schibilla H, Teunen D (Eds.). 1999. European Guidelines on quality criteria for computed tomography. European Commission, Office for Official Publications of the European Communities. EUR 16262 EN, Luxembourg. p. 70.

[13] BRYS P., (2017) Dosimétrie de trois examens TDM fréquemment prescrits en Belgique: étude multicentrique. COLLEGE DE RADIOLOGIE 2017.

[14] Beeres M., Römer M., Bodelle B., Lee C., Gruber-Rouh T., Mbalisike E., Kerl J. M., Wichmann J. L., Schulz B., Vogl T. J., Bauer R. W. (2014) Chest-abdomen-pelvis CT for staging in cancer patients: dose effectiveness and image quality using automated attenuation-based tube potential selection. Cancer Imaging. 14 (28). doi: 10.1186/s40644-014-0028-7.
[15] Sutton DG., McVey S. (2014) CT chest abdomen pelvis doses in Scotland: has the DRL had its day? Br J Radiol. DOI: $10.1259 /$ bjr.20140157.

[16] Esseghaier S, Ben Mabrouk A, Mrabet A, Daghfous MH. (2016). Évaluation de la dose-patient au scanner multicoupe dans un centre hospitalo-universitaire tunisien: étude de 250 patients adultes. Radioprotection 51 (4): 271-277.

[17] ICRP, (2007). International commission on radiological protection. Recommandations of the ICRP. IRCP Publication 103 Ann. ICRP 37 (2-4) Oxford, Pergamon Press.

[18] UNSCEAR, 2011. Rapport du Comité scientifique des Nations Unies pour l'étude des effets des rayonnements ionisants 2010, United Nations Scientific Committee on the Effects of Atomic Radiation. United Nations Publication; 2011.

[19] Pauwels EKJ, Bourguignon M. 2011. Cancer induction caused by radiation due to computed tomography: a critical note. Acta Radiol. 52 (7): 767-773.

[20] IRSN, (2016) Analyse des données relatives à la mise à jours des niveaux de référence diagnostique en radiologie et en médecine nucléaire, Bilan 2013-2015. 